\title{
Field Evaluation of Lethal Ovitraps for the Control of Dengue Vectors in Islamabad, Pakistan
}

\author{
Imrana Noreen ${ }^{1}$, Imtinan Akram Khan ${ }^{1, ~ *, ~ E m a d ~ K h a t e r ~}{ }^{2,5}$, Muhammad Naeem ${ }^{4}$, \\ Ahmad Mohammad Allam ${ }^{6}$, Rizwan Ahmed ${ }^{3}$, Muhammad Mohsin ${ }^{5}$, Soaib Ali Hassan', \\ Doaa E. Soliman ${ }^{7}$ \\ ${ }^{1}$ Department of Medical Entomology and Disease Vector Control, Health Services Academy, Islamabad, Pakistan \\ ${ }^{2}$ Department of Medical Entomology, Faculty of Science, Ain Shams University, Cairo, Egypt \\ ${ }^{3}$ Department of Entomology, University of Agriculture, Faisalabad, Pakistan \\ ${ }^{4}$ Department of Entomology, PMAS-Arid Agriculture University, Rawalpindi, Pakistan \\ ${ }^{5}$ Public Health Pests Laboratory of Jeddah Gov., Jeddah, Saudi Arabia \\ ${ }^{6}$ Department of Parasitology and Animal Diseases, Veterinary Research Division, National Research Centre, Cairo, Egypt \\ ${ }^{7}$ Department of Entomology, Ain Shams University, Abbassia, Cairo, Egypt
}

\section{Email address:}

imtinanakramkhan@yahoo.com (I. A. Khan)

${ }^{*}$ Corresponding author

\section{To cite this article:}

Imrana Noreen, Imtinan Akram Khan, Emad Khater, Muhammad Naeem, Ahmad Mohammad Allam, Rizwan Ahmed, Muhammad Mohsin, Soaib Ali Hassan, Doaa E. Soliman. Field Evaluation of Lethal Ovitraps for the Control of Dengue Vectors in Islamabad, Pakistan. International Journal of Ecotoxicology and Ecobiology. Vol. 2, No. 1, 2017, pp. 16-25. doi: 10.11648/j.ijee.20170201.13

Received: November 22, 2016; Accepted: December 14, 2016; Published: January 16, 2017

\begin{abstract}
Limited success has been attained using long-established mosquito vector control methods to prevent dengue transmission. Integrated disease control programs making use of alternative tools, e.g. Lethal ovitraps may provide greater prospects for monitoring and reducing vector populations and disease transmission in order to provide new robust data on the efficiency of entomological surveillance methods to control important dengue and other disease vectors in Pakistan and other geographic regions. The purpose of this study was to figure out the efficiency of Lethal ovitraps in eggs collection baited with grass infusion. This study also aimed at exploring Aedes infestation indices and generation of baseline data by indoor and outdoor ovi-trapping. Field evaluation of a Lethal ovitraps containing Deltamethrin-treated strip was carried out for monitoring the dengue vector (s) Aedes mosquitoes during November-February, 2015 in Rawal Town, Islamabad, Pakistan. The study site was divided into treatment and control blocks with 20 randomly selected houses for each block. Each block received 40 Lethal ovitraps (LOs) with and without treatment. The oviposition response by Aedes mosquitoes was measured using the Ovitrap Positive Index (OPI) and the Eggs Density Index (EDI). There were six weekly eggs collections made. Which yielded 510 Aedes eggs with 32 and 478 from the treatment and the control blocks, respectively, indicating the damaging effect of Deltamethrin on the treatment group. The weekly egg collections yielded 510 Aedes eggs with 32 and 478 from the treatment and the control blocks, respectively, indicating the damaging effect of Deltamethrin on the treatment group. The OPI response of treatment and control ovitraps was different. OPI was higher in the controls than in the treatment groups. Moreover, there was a significant difference in EDI of treatment compared to control. There was complete inhibition of larvae emergence in Lethal ovitraps in comparison to the control, where $50.20 \%$ larvae were formed. The results indicated that the Lethal ovitraps proved to be a very effective tool for monitoring and controlling Aedes populations under natural conditions. Furthermore, a significant decrease in the number of eggs was obtained in the treated group. At lower operational costs and consistency, these LOs can be practically used as a benign tool for measuring infestation rates for entomological surveillance of Aedes species.
\end{abstract}

Keywords: Aedes Aegypti, Aedes Albopictus, Lethal Ovitrap, Deltamethrin, Dengue 


\section{Introduction}

Dengue is the most rapidly spreading mosquito-borne viral disease in the world [1] that has proved damaging. The dengue incidence has increased 30-fold with an average rate of 50-100 million new infections / year in more than 100 countries (WHO, 2012) [2]. Approximately 2.5 billion people live in dengue endemic countries [3] along with 500,000 cases of dengue fever (DHF), resulting in around 24,000 deaths annually [4].

Dengue or dengue-like epidemics were reported throughout the nineteenth and early twentieth centuries all over the globe [5, 6] including Pakistan. In Pakistan, the dengue infections are becoming more frequent and severe in large cities [1]. Pakistan was first hit by dengue fever in 1994 while the worst epidemic was observed in 2011 in Punjab resulting in 203 deaths. The Disease Early Warning System (DEWS) in Pakistan reported 4,388 suspected cases from 01 January to 11 September 2013. The worst hit provinces were Khyber Pakhtunkha (3,177 cases) and Sindh (1098 cases) [7].

Domestic populations of the mosquito Ae. aegypti are the primary vectors of dengue (Lane \& Crosskey, 1993) [8], while Ae. albopictus is considered a secondary vector. Both species are sensitive to environmental conditions [9-12]. The dengue virus has also been isolated from species such as $A e$. albifasciatus, Ae. polinensis and several species of the Ae. scutellaris complex $[13,14]$. These mosquitoes primarily breed in artificial containers like earthen jars, plastic and metal drums, used car tyres, potted plants and man-hole covers and other types (Chareonviriyaphap et. al., 2003) [15].

Dengue virus is transmitted to humans through the bites of infective female Aedes mosquito [5, 6, 16]. Most countries in SouthEast Asia region (SEAR) bear a high burden of DF (Dengue fever)/DHF and experience frequent and cyclical epidemics [10, 17]. The dengue virus (DENV) belongs to the genus Flavivirus and has four antigenically distinct virus serotypes or genotypes (DENV I to DENV IV) (Westaway \& Blok, 1997) [10, 17, 18].

While a vaccine is under process, vector control remains the most effective and affordable method (Chandre, et. al., 1999; Nauen, 2007) [19] to prevent dengue transmission through integrated control approach including community participation. This requires a behavioural change about or toward the vector species and the disease [11] along with the tactical approach to target Aedes at their developmental stages in all settings where human-vector contact occurs [1].

The common methods used for Ae. aegypti surveillance include the inspection of premises for larvae and pupae and the use of ovitraps. Most operational surveillance systems depend on such surveys to gather their house, Breteau and larval-density indices [20], while others require source reduction through environmental sanitation or employ insecticide treatment (PAHO, 1994). It is not possible to eradicate diverse mosquito breeding sites completely. In addition, no adulticide or larvicide has proven fully successful against Aedes vectors (PAHO, 1994) [20, 21].

The Global Strategy for the Prevention and Control of DF and DHF also recommends the applications of integrated vector control measures with community and inter-sectoral involvement [22]. Therefore, in this regard, the development of a Lethal ovitrap (LO) as an alternative and emerging control technology [23], proved to be a practically cost-effective and suitable method for integrated vector control [20,21].

Ovitraps have been used to present useful data for Aedes control operations along with revealing low mosquito populations. Although ovitraps are used to attract mosquito females for eggs lying [20], they have many limitations as they themselves might become potential breeding places if not monitored regularly in less-than-a-week intervals [24].

The first ovitrap made in the United States has been used in many parts of the world for monitoring Ae. aegypti populations (Service, 1993). It was practically used against Ae. aegypti in 1969 at Singapore International Airport (Chan, 1973). Later, Chan et al., (1977) came up with a design of an autocidal screened ovitrap with greater efficacy in the field [8, 21, 25].

Addition of 'hay infusion' as an attractant (Reiter et. al., 1991) yielded higher number of eggs without altering its attractiveness regardless of seasonal variations [25]. Then, Zeichner and Perich $(8 \& 21)$ used an insecticide-treated oviposition strip in order to make the trap lethal to both larvae and adult Ae. aegypti. Based on these studies, an ovitrap can be modified for controlling Aedes vectors. Therefore, we decided to use an insecticide-treated ovistrip to be evaluated against Aedes in a field trial [8].

The LO using $25 \%$ wettable granulated Deltamethrin is being described for the first time in Pakistan. These ovitraps collect mosquito immature stages for various research purposes and serve as suitable surveillance and monitoring tools. Not much work has been done on LOs except their use in Lahore by Jahan N. [15, 17, 19, 25]. Realizing the need of time, this study was planned to use LOs for reducing vector density sufficiently in an area as a part of integrated vector management strategy. An additional advantage of using LOs is to shorten the longevity of the vector, which ultimately reduces the number of infective mosquitoes in natural populations with the long-term implications for the mosquito vectorial capacity.

The overall aim of this study was to decrease the disease burden of dengue by using economical and benign surveillance tools for monitoring dengue vectors while the objectives fulfilled were; 1) to compare the efficiency of Lethal ovitraps in eggs collection baited with $10 \%$ grass infusion and water solution against standard ovitraps, 2) to explore the relationship between different Aedes infestation indices [26], \& 3) to generate baseline data about infestation by evaluating indoor and outdoor ovitraps.

\section{Materials and Methods}

\subsection{Study Design and Populations}

This study was Clustered-Randomized (Winer et. al., 1991) in which a group of 20 houses was used as a treatment block, while another group of 20 houses was used as a control block.

\subsection{Study Area}

The study was carried out in Rawal town of Islamabad, 
Pakistan during November 2014-February, 2015. Islamabad $\left(33^{\circ} 43^{\prime} \mathrm{N} / 73^{\circ} 04^{\prime} \mathrm{E}\right)$ and has an area of $906 \mathrm{~km}^{2}$ [27] with a human population of 8052035 (census 1998). Rawal town $(33.685 \mathrm{~N} / 73.117 \mathrm{E})$ of Islamabad district has a population of 17,292 people (census 1998).

Islamabad is a part of a semi-arid-sub-tropical climate zone with a large variation in temperature. The mercury here sometimes falls below $0^{\circ} \mathrm{C}$ during winter and sometime touches $48^{\circ} \mathrm{C}$ during summer. The average rainfall ranges from 990 to $1000 \mathrm{~mm}$. Thunder, wind and hailstorm are also common [28].

\subsection{Dengue Mosquito Vectors Surveillance}

During a preliminary survey of two weeks, an adults collection was made using mechanical aspiration [29] collecting mosquitoes within the house for 10 minutes [21] during morning hours (0700-0900 hrs) [30]. The collections were made both inside and outside the selected houses. The captured adult mosquitoes were placed in entomological boxes and brought back to the laboratory for species identification [29] using standard identification keys [30].

In addition, mosquito larvae and pupae were collected from natural containers using larva-fishing nets. The contents collected were transferred to a beaker containing water and transported to the entomological laboratory for species identification [31]. Conventional indices i.e. the House (HI), the Container (CI), the Breteau (BI) and the Pupal (PI), were calculated according to the WHO guidelines [16, 32]. After intervention of six weeks, a post survey was also carried out for collecting mosquito adults, larvae and pupae in the last two weeks of the study.

\subsection{Ovitrap and Ovipaddle Design}

LOs were used as a lure-and-kill device for dengue vectors using illustrations of Fay and Perry (1965) [33], consisting of $500 \mathrm{ml}$ capacity black-painted plastic cups. Two holes were drilled equidistant at $2 \mathrm{~cm}$ below the cup rim to hang the ovitrap using a 50-cm string [25]. A wooden tongue depressor $(16 \mathrm{~cm} \times 2.5 \mathrm{~cm})$ [21] wrapped by layers of filter paper served as the ovistrip. The ovistrips were treated with $1.0 \mathrm{mg}$ active ingredient/strip of $25 \%$ wettable granulated deltamethrin [34, 35] found to be most effective in prior laboratory testing (Zeichner \& Perich, 1999) [8, 21]. Ovistrips were pre-treated with insecticide solution pipetted evenly over the paper strip and were left to dry to be ready for use [8].

To enhance the attractiveness of the LOs, they were filled with a $10 \%$ hay infusion-water solution as described by Reiter et al., (1991) to approximately $2 \mathrm{~cm}$ of the top [21]. Hay infusion was made by steeping $125 \mathrm{~g}$ of dried lawn grass in 15 liters of tap water in a tightly-closed plastic garbage container and incubated for seven days [36].

\subsection{Ovitrap Placement}

The selected area was divided into two blocks separated by 100-200 m distance. One block of 20 houses was randomly assigned as a control block and other as a treatment block. For all experiments, ovitraps were set between 0900 and 1200 hours, the time of least oviposition activity (Chadee and Corbet 1987, Gomes et. al., 2005) [37].

Each house received 2 ovitraps, one indoor and other outdoor placed at the height of 1.5-2 $\mathrm{m}$ [38]. These traps were set sheltered from direct sunlight and rain.

\subsection{Evaluation of LETHAL Ovitraps}

Ovitraps without any treatment containing $10 \%$ hay infusion and water solution were installed in 20 houses (1 pair for each house) of control block alternatively while lethal ovitraps with deltamethrin $25 \%$ wettable granulated ( $1 \mathrm{mg} / \mathrm{strip})$ in $10 \%$ hay infusion and water solution were installed in 20 houses ( 1 pair for each house) of the treatment block. All ovitraps $(\mathrm{n}=80)$ were observed weekly for Aedes eggs and $10 \%$ hay infusion and water solution was replaced in respective ovitrap. Ovipaddle or entire ovitrap was replaced if anyone was found missing. To prevent fungal contamination, all ovipaddles were replaced with fresh ovipaddles weekly.

From collection site, each of one week-old ovipaddle was brought carefully at the entomological insectary after placing in an individual labeled plastic bag. The eggs on pallets were counted using a stereoscopic microscope [31]. Ovitraps were considered positive when at least one egg was detected [39]. Each ovipaddle was left to dry at room temperature $\left(28 \pm 2{ }^{\circ} \mathrm{C}\right.$; $80 \pm 10 \% \mathrm{RH})$ at diagonal angle [25]. They were maintained in laboratory conditions until embryonic development was complete [31]. Identification of the species was carried out after adult stage [40] using Zoo taxa keys (Rueda, 2004) [41].

The total dried filter paper strips were shifted to the respective larval tray filled with sufficient amount of tap water to allow egg eclosion. The larval trays were labeled and covered with a net to block intervention. In each tray larvae formed were counted and recorded. Pupae were counted and separated a separate dropper (for each treatment) to a cup containing $200 \mathrm{ml}$ of water for adult emergence. In each cup, the adults emerged were counted and recorded. Mortality in each stage was recorded daily, was and counted by separating dead larvae or pupae from live ones with using camel hair brush. No food was added during the whole experiment period. Water was daily added to balance the water loss by evaporation [25]. Weekly temperature and accumulated rainfall was also taken into consideration during the studied period.

\subsection{Data Entry and Analysis}

The data collected were analyzed in SPSS (Statistical Package for Social Sciences) version 16.

The Ovitrap Positivity Index (OPI) and the Eggs Density Index (EDI) were used as indicators of oviposition level. These indices were calculated on a weekly basis (Gomes, 1998). The mean numbers of eggs were calculated for the total trial period and also for individual weeks according to the ovitrap installation blocks. The results were also 
calculated with reference to percentage emergence and SEM (Standard error of the mean). Where required, cross tabulation was done and association was seen by checking the significance of the association using Chi-square statistical test and by applying ANOVA (Analysis of Variance).

Infestation by Aedes eggs was estimated using conventional indicators; OPI and EDI (Gomes, 1998) [24, 25]. Choice of Lethal ovitrap for oviposition (OPI) was estimated as the percent Lethal ovitraps positive for eggs from the total number of ovitraps inspected (or distributed).

OPI $=$ No. of Lethal ovitraps positive for eggs / Total no. of Lethal ovitraps inspected $\times 100$

The efficiency of Lethal ovitraps in eggs collection (EDI) was calculated as average number of eggs laid per positive control/Lethal ovipaddle.

$\mathrm{EDI}=$ Total no. of Aedes eggs on ovipaddles / No. of positive ovitraps

To evaluate the toxic effect of Lethal ovitraps after field exposure, percent larvae, pupae and adults emergence was calculated. Test results compared with their respective controls were statistically analyzed using Excel and SPSS version 16.0; SPSS Inc., Chicago, IL)

\section{Results}

The studied period was characterized by a mean monthly temperature of $72.2^{\circ} \mathrm{F}\left(62.4-81.5^{\circ} \mathrm{F}\right)$ and rainfall of 12.93 inches (11.9-13.9 inches).

\subsection{Adults Density}

Six weeks after placing LO, the number of female Aedes collected was notably fewer from the treatment houses with only five mosquitoes aspirated in comparison to 13 mosquitoes from the controls. This indicates that the LO over time significantly reduced adult female Aedes production. The mean number of female Aedes aspirated from the treatment houses $(0.25)$ compared to the mean number collected from the control houses $(0.65)$ was not distinctively different.

Although there was no change in the mean of pretreatment $(0.25)$ to the mean number in post-treatment $(0.25)$ in treatment block, but at least, this value did not elevate. This might be due to the presence of a large number of breeding places available competing with the LO for the oviposition-seeking Aedes females. This result is consistant with the study of Perich et al., in Brazil at Nilopolis. The overall mean of both the blocks was 0.2875 . This manifests a significant result $(\mathrm{P}<0.05) \mathrm{P}=0.022$

\subsection{Prevalence Indices of Aedes Species}

The four indices indicated different levels of infestation by Ae. aegypti and Ae. albopictus. HI and BI registered identical values in treatment blocks before and after the study started. In total, 80 houses were searched for Aedes breeding. Aedes breeding was only detected in 5 houses. About 411 water containers were searched, out of which only 9 were found positive for Aedes breeding. During a preliminary survey, the $\mathrm{HI}$, the CI, the BI and the PI of the treatment block were found to be $5,0.8,5$ and zero, respectively. There was no larvae or pupae found during a preliminary survey in the control block. After treatment of six weeks, the HI, the CI, the $\mathrm{BI}$ and the PI of the treatment block remained 5, 1.06, 5 and 0.01 , respectively in comparison to the $\mathrm{HI}$, the $\mathrm{CI}$, the $\mathrm{BI}$ and the PI of the control block. (Figure 1)

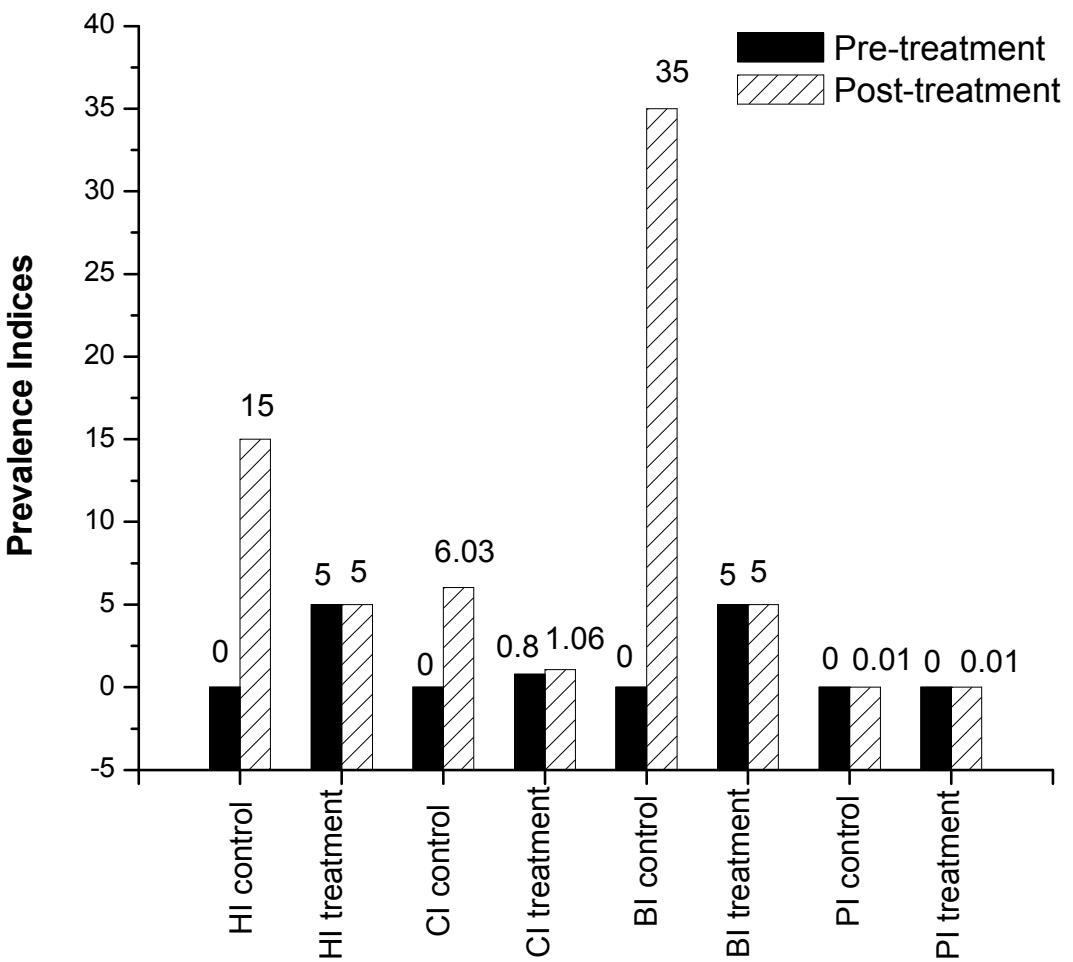

Figure 1. Graph showing effect of treatment on prevalence indices of Aedes aegypti and Ae. Albopictus. 
The CI of the treatment block (1.06) after the treatment was significantly different compared to the pre-treatment $(0.8)$ of the treatment block rather it was higher. This may be linked to peak activity period of the mosquitoes (Figure 1).

\subsection{Eggs Density}

During the four months of the experiment, a total of 510 eggs of Ae. albopictus and Ae. aegypti were collected in the ovitrap out of 480 containers for both blocks. Eggs were collected for 6 weeks, while a very small number of eggs were collected throughout the collection time period.

\subsection{Ovitrap Positive Index and Egg Density Index}

Out of 480 containers, only 41 were found positive for Aedes eggs. Average 15.93 eggs were harvested per ovipaddle in the control block, while 2.90 eggs were obtained per ovipaddle from treatment block. Collections of eggs differed between two blocks chosen, which gave the OPI of 8.54 while EDI was 12.439 (Table 1). OPI and EDI were also calculated for each week per block (Figure 2).

Table 1. Eggs obtained in Rawal town.

\begin{tabular}{|c|c|c|c|c|c|c|c|c|c|c|c|c|}
\hline & & \multicolumn{3}{|c|}{ No. of LOs } & \multicolumn{2}{|c|}{ Positive LOs } & \multirow{3}{*}{$\begin{array}{l}\text { Total } \\
\text { no of } \\
\text { eggs }\end{array}$} & \multirow{3}{*}{$\begin{array}{l}\text { \% of } \\
\text { Total } \\
\text { Sum }\end{array}$} & \multicolumn{4}{|c|}{ Eggs/LO } \\
\hline & & \multirow{2}{*}{ Installed } & \multirow{2}{*}{$\begin{array}{l}\text { Collections } \\
\text { (n) }\end{array}$} & \multirow{2}{*}{$\begin{array}{l}\text { Total } \\
\text { (n) }\end{array}$} & \multirow{2}{*}{ (n) } & \multirow{2}{*}{ OPI } & & & \multirow{2}{*}{ EDI } & \multirow{2}{*}{ \pm SEM } & \multicolumn{2}{|c|}{ 95\% Confidence Interval for Mean } \\
\hline & & & & & & & & & & & Lower Bound & Upper Bound \\
\hline \multirow{3}{*}{ Blocks } & Control & 40 & 6 & 240 & 30 & 12.5 & 478 & $93.70 \%$ & 15.93 & 3.103 & 9.58 & 22.28 \\
\hline & Treatment & 40 & 6 & 240 & 11 & 4.583 & 32 & $6.30 \%$ & 2.9 & 0.638 & 1.48 & 4.33 \\
\hline & Total & 80 & 12 & 480 & 41 & 8.542 & 510 & $100.00 \%$ & 12.43 & 2.443 & 7.5 & 17.37 \\
\hline \multirow{3}{*}{$\begin{array}{l}\text { Position } \\
\text { of the } \\
\text { ovitraps }\end{array}$} & Indoor ovitraps & 40 & 6 & 240 & 7 & 2.91 & 37 & $7.30 \%$ & 5.28 & 3.307 & 2.8 & 13.37 \\
\hline & Outdoor ovitraps & 40 & 6 & 240 & 34 & 14.16 & 473 & $92.70 \%$ & 13.91 & 2.815 & 8.18 & 19.64 \\
\hline & Total & 80 & 12 & 480 & 41 & 17.08 & 510 & $100.00 \%$ & 12.43 & 2.443 & 7.5 & 17.37 \\
\hline
\end{tabular}

There was a significant difference $(\mathrm{P}<0.05) \mathrm{P}=0.016$ between two blocks in terms of the number of eggs, OPI and EDI.

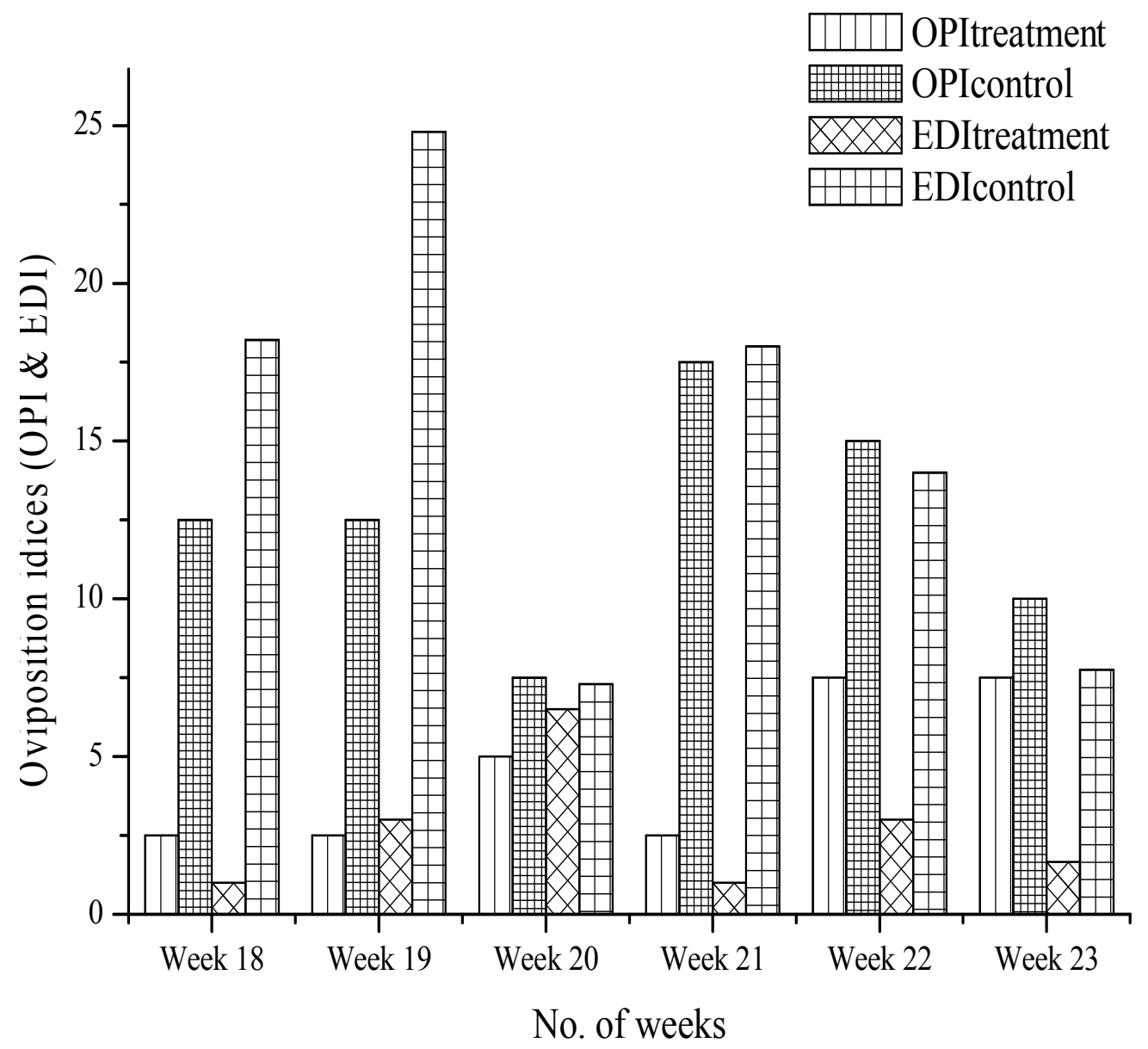

Figure 2. Graph showing OPI and EDI indices of treatment and control block. 
There were significant differences in the presence and density of eggs between outdoor and indoor ovitraps with higher values for outdoor ovitraps (OPI=14.16; EDI = 13.91) (Figure 3). The gravid female Aedes mosquitoes had a significant preference to oviposit eggs in ovitraps placed outside $(\mathrm{F}=1.8$; df $139 ; \mathrm{P}=0.187)$.
Only one outdoor ovitrap was positive for mixed breeding which accounted for $2.9 \%$ of the total 34 outdoor ovitraps collected. Two species were identified after adult emergence as Ae. aegypti and Ae. albopictus. However, there was no mixed breeding found in ovitrap surveillance in treatment.

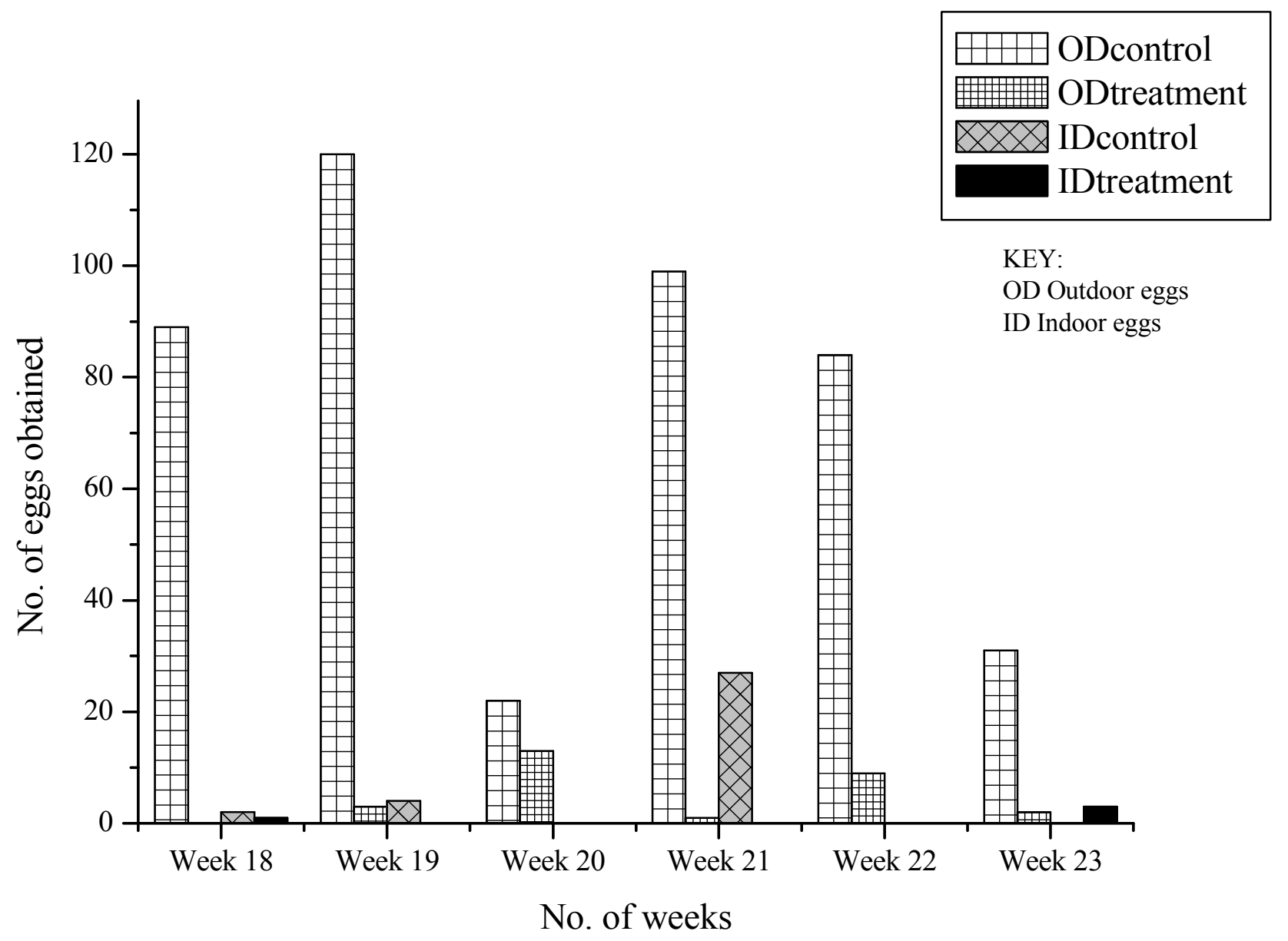

Figure 3. Graph showing indoor and outdoor oviposition preference of Aedes.

\subsection{Percentage Emergence Rate}

Ovitraps collected the largest number of Ae. albopictus eggs. Only Ae. aegypti eggs were obtained in entomological week no. 18. Out of 510 eggs, 240 larvae hatched (47.05\%) of which 5 five were Ae aegypti (2.08\%) and the rest (97.91) were Ae. albopictus (\%). Percent larvae and pupae formation along with adult emergence was reduced to zero in treatment block as compared to control. (Figure 4)

The control ovitraps yielded 50.20\% larvae, 90\% pupae and $98.61 \%$ adult. Overall treatment group was found to be most effective in controlling adult population of Aedes in the selected locality.

\subsection{Species Identification of Emerged Adults}

Generally, Ae. albopictus was found at a higher frequency than Ae. aegypti in these ovitraps.

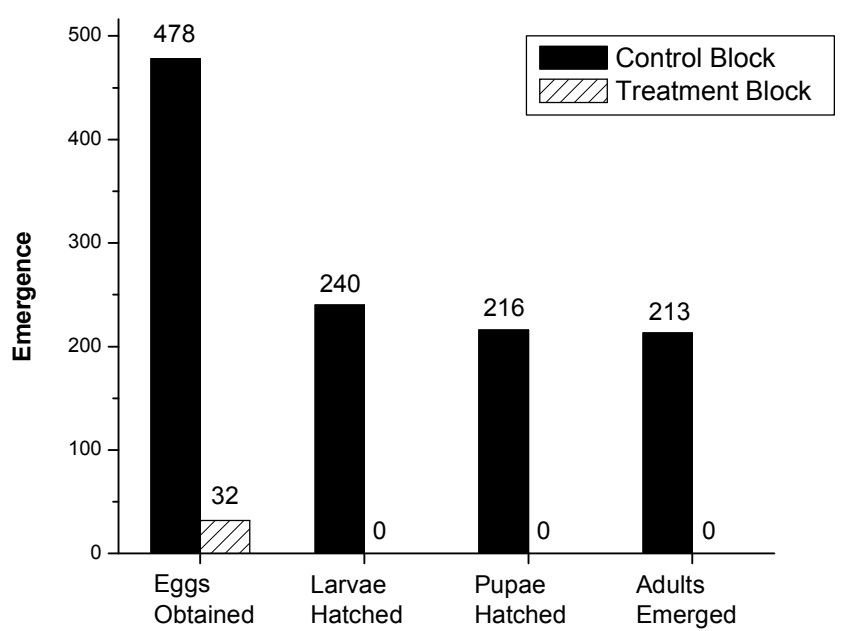

Figure 4. Graph showing the emergence rates of various developmental stages. 
Out of 213 adults emerged, 208 (97.65\%) were Ae. albopictus, and only five $(2.34 \%)$ were identified as $A e$. Aegypti. The overall mean for both species was 5.19. None of the eggs hatched in the treatment group. (Figure 4) Therefore, no adult identification was possible for the treatment block.

There were significant differences in the presence of $A e$. albopictus compared to Ae. aegypti $(\mathrm{F}=2.265$; df 3 37; $\mathrm{P}=$ 0.097), with Ae. albopictus was the predominant species.

\section{Discussion}

The infestation indices indicated the presence of Aedes species during the study period in Rawal Town, Islamabad. The LOs did effectively compete with the other domestic containers for oviposition as they reduced the number of positive containers (Figure 2). The results of our study are consistent to the results of Chan et. al., (1977) and Perich et. al., [21]. The PI values recorded were above $1 \%$ and $\mathrm{BI}$ above $5 \%$, indicate the risk areas where dengue transmission is likely to occur (FUNASA, 2002).

In the present study, the four larval surveys conducted showed a positive correlation between $\mathrm{HI}$ and BI. (Figure 1) Therefore, the larval indices recorded from the study area indicate that such high index $(\mathrm{HI}=15 \%)$ may lead to an outbreak if no immediate actions are taken. For the index of pupa per person (Focks et al., 1995), our results indicated the value of 0.01 where dengue vector remains unable to sustain dengue transmission.

There are almost no control actions taken by the municipalities to control potential dengue vectors. From the positivity of the ovitraps and the number of eggs deposited throughout the study period, it can be assumed that female Aedes were constantly multiplying. Therefore it indicates the urgent demand of at least reducing the mosquito number from attaining the threshold toward the increased risk of dengue transmission.

The presence of Aedes females, but not of larvae, indicated fewer breeding places for larvae. The positivity of the studied area in terms of EDI and negativity in terms of HI, CI, BI and PI indicates the accuracy of ovi-trapping at measuring infestation level of Aedes. We observed in our study that despite poor prevalence indices recorded, ovitrap surveillance produced quantitative results (Figure 2). Similar results were reported $[31,42]$. These studies considered LOs strategy as an effective tool to detect and prevent Ae. aegypti population growth, which lowers the risk of potential disease outbreaks.

The current study evaluated LOs impregnated with deltamethrin. It was found that the treated ovitraps received significantly lower number of Aedes eggs as compared to their respective control groups (Figure 2 and 3). The effect of deltamethrin impregnated LOs was also studied in Thailand (Sithiprasasna, et. al., 2003) and Brazil to observe Ae. aegypti population (Perich et. al., 2003) [21]. Deltamethrintreated ovitraps used in Manawann, Lahore [25] also indicated that LOs containing 1.5\% EC deltamethrin could serve as an effective dengue vectors suppression tool but the control of mosquito vector will only be attained if it is used effectively as a part of an IVM programme. Perich et. al., [21] \& Jahan N, Sarwar MS [25] also suggested the incorporation of LOs in integrated disease control programs.

We found that black ovitraps come with good yield for the surveillance of gravid Ae. albopictus, but not for Ae. aegypti as only five mosquitoes of this species were found. Similar results were depicted by in North-Central Florida, USA [40]. We also found the mixed breeding of Ae. aegypti and Ae. albopictus in our study where Ae. albopictus were dominant compared to Ae. aegypti. Sulaiman et. al., (1991), Norzahira et. al., Rozilawati et. al., Shi CH, Sallehudin et. al. and Chen et. al., [38] also reported similar results.

We also found that that Ae. albopictus preferred to breed outdoors (473 eggs, mean/trap or collection site \pm SEM)) rather than indoors (37 eggs) in Rawal Town, Islamabad (Table 1). This was also found by Rozilawati et. al., \& Chen et. al., \& Dibo et. al., [38, 42]. This may explain the preference of Ae. albopictus to breed outdoors rather than indoors.

The positive correlation between OPI and EDI (Table 1) found for both indoor and outdoor placement of the ovitrap is in agreement with that reported by Chadee (1992), Dibo et. al., (2005) [42] \& Burroni et. al., (2013) [39]. Although there was a large number of natural containers present in the studied area for breeding but some traps became positive within the first week, that is, during the dry season. Thus, this method was confirmed to be efficient in detecting of Aedes (Stegomyia) as has been described by Fay and Eliason (1966), Marques et. al., (1993), Braga et. al., (2000) \& Dibo et. al., (2005) [42].

The geographical distribution of Aedes in Rawal Town, Islamabad gave only a rough estimate of the infestation, which cannot be applied to the entire geographical region of Islamabad. The number of eggs harvested was very less due to commencement of the study in early dry season in the area and also we faced the problem of closed houses.

\section{Conclusion}

The LO placed in Rawal Town had a significant damaging effect on Aedes populations within three months after their placement inside and outside the treatment houses. In comparison of two blocks, the LOs in the control block harvested eggs about 14.9 times the numbers harvested by the treatment block.

Lethal ovitraps were found most effective tool as it directly targeted dengue vectors with minimal use of insecticides. Furthermore, the minimum effective dosages to inhibit $100 \%$ larval emergence and subsequent stages in a habitat have shown to be extremely low (1 mg/ovistrip).

Mixed breeding indicates that more than one mosquito species can oviposit in a single ovitrap. Therefore it can be assumed from our study and other cited that LO is highly sensitive to attract gravid females of more than one mosquito species to oviposit in the container. 
Based on the data acquired from LO surveillance, it is concluded that LOs are useful in planning anti-Aedes campaign, insecticide application and use of new technologies for vector control. Its effectivity in Aedes control demands IVM by using a set of effective interventions rather than sole reliance on LOs. For a country like Pakistan, LOs are more practical surveillance tools as a useful indicator for assessing the impact of control programmes as they are inexpensive, simple to handle and non-disturbing.

\section{References}

[1] World Health Organization (WHO), Research SPf, Diseases TiT, Diseases WHODoCoNT, Epidemic WHODo, P. Alert, "Dengue guidelines for diagnosis, treatment, prevention and control," World Health Organization, 2009, pp. 3-59.

[2] World Health Organization (WHO), "Global strategy for dengue prevention and control 2012-2020.” ISBN 978924 1504034 .

[3] World Health Organization (WHO), Dengue \& dengue haemorrhagic fever, Factsheet No 117, revised May 2008, Geneva, pp. 25-8.

[4] F. Jahan "Dengue fever (DF) in Pakistan," Asia Pac. Fam. Med. 2011; vol. 10 (1): pp. 1.

[5] World Health Organization (WHO), "Dengue haemorrhagic fever: diagnosis, treatment, prevention and control," World Health Organization, Geneva, Switzerland. 2nd ed. 1997, 1.

[6] World Health Organization (WHO). "Leishmaniasis and leishmania/HIV co-infection WHO report on global surveillance of epidemic-prone infectious diseases," 2000. Report no. WHO/CDS/CSR/ISR/2000.1. Geneva, The Organization, 2000, pp. 75-7.

[7] World Health Organization (WHO). "The weekly epidemiological monitor" WHO, Regional office for the Eastern Mediterranean, Cairo, Egypt, 2013, vol. 6 (37).

[8] B. Zeichner, \& M. Perich, "Laboratory testing of a lethal ovitrap for Aedes aegypti," Medical and veterinary entomology, 1999, vol. 13 (3), pp. 234-8.

[9] R. Bhatia, AP. Dash, \& T. Sunyoto, "Changing epidemiology of dengue in South-East Asia," WHO South-East Asia Journal of Public Health, 2013, vol. 2 (1), pp. 23.

[10] D. J. Gubler, "Dengue and dengue hemorrhagic fever," Clinical microbiology reviews, 1998, vol. 11 (3), pp. 480-96.

[11] J. G. Rigau-Pérez, G. G. Clark, D. J. Gubler, P. Reiter, E. J. Sanders, \& A. V. Vorndam, "Dengue and dengue haemorrhagic fever," The Lancet., 1998, vol. 352 (9132), pp. 971-7.

[12] A. C. Moncayo, Z. Fernandez, D. Ortiz, M. Diallo, A. Sall, \& S. Hartman, et al, "Dengue emergence and adaptation to peridomestic mosquitoes," Emerg. Infect Dis., 2004, vol. 10 (10), pp. 1790-6.

[13] R. A. Martínez-Vega, R. Danis-Lozano, J. Velasco-Hernández, F. A. Díaz-Quijano, M. González-Fernández, \& R. Santos, et al., "A prospective cohort study to evaluate peridomestic infection as a determinant of dengue transmission," Protocol, BMC public health, 2012, vol. 12 (1), pp. 262.

[14] K. B. Chua, I. Chua, I. Chua, \& K. H. Chua, "Differential environmental preferences of gravid female Aedes mosquitoes in ovipositing their eggs," Southeast Asian journal of tropical medicine and public health, 2005, vol. 36 (5), pp. 1132.

[15] N. Jahan, \& N. Mumtaz, "Evaluation of resistance against deltamethrin in Aedes mosquitoes from Lahore, Pakistan, Biología (Pakistan)," 2010, vol. 56 (1 \& 2), pp. 9-15.

[16] World Health Organization, "Comprehensive guidelines for prevention and control of dengue and dengue haemorrhagic fever," 2011.

[17] N. Jahan, \& N. Mumtaz, "Evaluation of resistance against deltamethrin in Aedes mosquitoes from Lahore, Pakistan. Biología (Pakistan)," 2010, vol. 56 (1 \& 2), pp. 9-15.

[18] World Health Organization, "Practical manual and guideline for dengue vector surveillance World Health Organization," Medical research institute and dengue coordination unit, Sri Lanka, 2011, pp. 1-65.

[19] N. Jahan, M. S. Sarwar, \& T. Riaz, "Field evaluation of lethal ovitraps impregnated with deltamethrin against dengue vectors in Lahore, Pakistan, Biological society of Pakistan," 2011, vol. 57 (1 \& 2), pp. 7-13.

[20] C. S. Tang, S. Lam-Phuab, Y. Chunga, \& A. Gigerc, "Evaluation of a grass infusion-baited autocidal ovitrap for the monitoring of Aedes aegypti (L.)," Dengue Bulletin, 2007, vol. 3, pp. 131-40.

[21] M. Perich, A. Kardec, I. Braga, I. Portal, R. Burge, \& B. Zeichner, et al., "Field evaluation of a lethal ovitrap against dengue vectors in Brazil," Medical and veterinary entomology, 2003, vol. 17 (2), pp. 205-10.

[22] E. Renganathan, W. Parks, L. Lloyd, M. Nathan, E. Hosein, \& A. Odugleh, et al., "Towards sustaining behavioural impact in dengue prevention and control," Dengue Bulletin, 2003, vol. 27, pp. 6-12.

[23] J. Entwistle, W. Robinson, \& A. de Carvalho Campos, "Emerging technologies for control of Aedes Aegypti and Aedes Albopictus (Diptera, Culicidae)," International Pest Control, 2011, vol. 53 (6), pp. 318.

[24] S. Santos, M. Melo-Santos, L. Regis, \& C. Albuquerque, "Field evaluation of ovitraps consociated with grass infusion and Bacillus thuringiensis var. israelensis to determine oviposition rates of Aedes aegypti," Dengue Bulletin, 2003, vol. 27, pp. 156-62.

[25] N. Jahan, \& M. S. Sarwar, "Field Evaluation of Lethal Ovitraps for the Control of Dengue Vectors in Lahore, Pakistan,” Pakistan J. Zool., 2013, vol. 45 (2), pp. 305-15.

[26] P. M. Saide, A. Che-Mendoza, E. A. Rebollar-Téllez, P. Coleman, \& C. Davies, "Field evaluation of traditional vs. baited ovitraps, single and paired, with different concentrations and ages of Bermuda grass for the surveillance of Aedes aegypti," London School of Hygiene and Tropical Medicine, 2003, pp. 1-23.

[27] Pakistan. Islamabad developmental authority, Islamabad, Available at www.rda.gov.pk. 
[28] Pakistan. Islamabad and Islamabad, "Multi Hazard Risk Mapping- Rural Development Policy Institute (RDPI), Islamabad," Available at www.rdpi.org.pk.

[29] M. R. Dibo, A. P. Chierotti, M. S. Ferrari, A. L. Mendonça, \& F. Chiaravalloti Neto, "Study of the relationship between Aedes (Stegomyia) aegypti egg and adult densities, dengue fever and climate in Mirassol, state of São Paulo, Brazil," Memorias do Instituto Oswaldo Cruz., 2008, vol. 103 (6), pp. 554-60.

[30] R. Singh, M. Das, R. Dhiman, P. Mittal, \& A. Sinha, "Preliminary investigation of dengue vectors in Ranchi, India," Journal of vector borne diseases, 2008, vol. 45 (2), pp. 170.

[31] V. C. Morato, Md. G. Teixeira, A. C. Gomes, D. P. Bergamaschi, \& M. L. Barreto, "Infestation of Aedes aegypti estimated by oviposition traps in Brazil," Revista de Saúde Pública, 2005, vol. 39 (4), pp. 553-8.

[32] R. T. I., "Training manual on malaria entomology, For entomology and vector control technicians (Basic Level)," Research Triangle Institute, 2012, pp. 76-78.

[33] R. Fay, \& A. Perry, "Laboratory studies of ovipositional preferences of Aedes aegypti," Mosquito News, 1965, vol. 25 (3), pp. 276-81.

[34] R. Nauen, "Insecticide resistance in disease vectors of public health importance," Pest management science, 2007, vol. 63 (7), pp. 628-33.

[35] World Health Organization (WHO), "Specifications and Evaluations for Public Health Pesticides: Deltamethrin. Geneva, Switzerland,” World Health Organization, 2008.

[36] K. A. Polson, C. Curtis, C. M. Seng, J. G. Olson, N. Chantha,
\& S. Rawlins, "The use of ovitrap baited with hay infusion as a surveillance tool for Aedes aegypti mosquitoes in Cambodia," Dengue Bull., 2002, vol. 26, pp. 178-84.

[37] A. L. Sant'ana, R. A. Roque, \& A. E. Eiras, "Characteristics of grass infusions as oviposition attractants to Aedes (Stegomyia) (Diptera: Culicidae)," Journal of medical entomology, 2006, vol. 43 (2), pp. 214-20.

[38] C. Chen, W. Nazni, H. Lee, B. Seleena, S. Mohd Masri, \& Y. Chiang, et al., "Mixed breeding of Aedes aegypti (L.) and Aedes albopictus Skuse in four dengue endemic areas in Kuala Lumpur and Selangor, Malaysia," Tropical biomedicine, 2006, vol. 23 (2), pp. 224-7.

[39] N. Burroni, V. Loetti, P. Prunella, \& N. Schweigmann, "Ovitraps placed in dwellings and on public paved areas for Aedes aegypti (Diptera: Culicidae) monitoring," Revista Colombiana de Entomología, 2013, vol. 39 (1), pp. 56-60.

[40] D. F. Hoel, P. J. Obenauer, M. Clark, R. Smith, T. H. Hughes, \& R. T. Larson et al., "Efficacy of Ovitrap Colors and Patterns for Attracting Aedes albopictus at Suburban Field Sites in North-Central Florida 1," J. Am. Mosq. Control Assoc., 2011, vol. 27 (3), pp. 245-51.

[41] L. M. Rueda, "Pictorial keys for the identification of mosquitoes (Diptera: Culicidae) associated with dengue virus transmission," DTIC Document, 2004.

[42] M. R. Dibo, F. Chiaravalloti-Neto, M. Battigaglia, A. Mondini, E. A. Favaro, \& A. A. Barbosa, et al., "Identification of the best ovitrap installation sites for gravid Aedes (Stegomyia) aegypti in residences in Mirassol, state of São Paulo, Brazil," Memórias do Instituto Oswaldo Cruz, 2005, vol. 100 (4), pp. 339-43. 